\title{
Alcohol as an independent risk factor for obstructive sleep apnea
}

\author{
Shanshan Yang ${ }^{1}\left[\right.$ Xinhong Guo $^{2} \cdot$ Wei Liu $^{3} \cdot$ Yanhua Li ${ }^{2} \cdot$ Yunxi Liu ${ }^{1}$
}

Received: 2 February 2021 / Accepted: 28 May 2021 / Published online: 10 June 2021

(c) The Author(s) 2021

\begin{abstract}
Background To determine if alcohol consumption is a risk factor for obstructive sleep apnea (OSA) and nocturnal oxygen desaturation.

Methods This case-control study evaluated patients with confirmed OSA and a control group using polysomnography (PSG). Two doctors who have worked in this field for more than 5 years provided a blinded interpretation of the patients' monitoring results. Logistic regression models were used to identify the odds ratio (OR) for alcohol consumption on OSA. Results A total of 793 patients were enrolled in this study. Compared with those who did not consume alcohol, those consuming alcohol had a higher risk of OSA (OR 2.03, 95\% CI 1.30-3.17) after adjustment. Regarding the risk of OSA after adjusting for former drinkers and current ones, the ORs were 1.96 (95\% CI 1.19-3.22) and 2.22 (95\% CI 1.06-4.63), respectively. And the $P$ for trend $=0.002$. The $\beta$ of former drinkers and the current ones were 3.448 and 4.560 after adjustment; $P$ for trend was 0.006. The relationship may have gender difference, and alcohol consumption was associated with AHI in female significantly ( $\beta=10.190$ and 15.395 for former and current drinkers, respectively, in females after adjustment, $P$ for trend $=0.002)$. Conclusions In this study, we found that alcohol consumption was an independent risk factor of OSA and OSA with hypoxia, and alcohol consumption was related to AHI significantly after adjustment, especially in female. In order to reduce the risk and severity of OSA, it is suggested that people should avoid drinking, and drinkers should abstain from drinking.
\end{abstract}

Keywords Alcohol drinking · Case-control study · Conventional cardiovascular risk factors · Obstructive sleep apnea

\section{Introduction}

Obstructive sleep apnea (OSA) is characterized by repetitive episodes of complete and partial obstructions of the upper airway during sleep. Previous studies [1] reported a prevalence of OSA in the general population of $2-10 \%$, and more men were affected than women, but the prevalence of

Shanshan Yang, Xinhong Guo, and Wei Liu contributed equally to this work.

Yanhua Li

13681061699@163.com

Yunxi Liu

1425628298@qq.com

1 Department of Disease Prevention and Control, Chinese PLA General Hospital, The 1st Medical Center, Beijing 100853, China

2 Department of Cardiology, Chinese PLA General Hospital, The 1st Medical Center, Beijing 1000853, China

3 Chinese PLA General Hospital, Hospital Management Institute, Beijing, China
OSA was as high as $50 \%$ in patients with CHD and in those with metabolic syndrome [2]. In recent years, the prevalence of OSA has shown an increasing trend. OSA patients who experience hypoxia during sleep and are not treated for a long time may experience a variety of serious cardiovascular and cerebrovascular diseases, and even sudden death at night [3,4]. A recent study by Franklin et al. [5] showed that OSA was present in $22 \%$ of male patients and $17 \%$ of female patients; women with OSA were more likely to develop hypertension, obesity, and other cardiovascular and cerebrovascular diseases that are age-related; and women with OSA may have clinical manifestations that differ from those of men, making the diagnosis more difficult. Obesity is a major risk factor for snoring and sleep apnea, but Franklin et al. reported that $39 \%$ of patients with OSA were of normal weight. Age and obesity are recognized risk factors for OSA. Other risk factors, such as the relationship between smoking and drinking, and sleep apnea remain contradictory. Foreign studies suggest that smoking and drinking are not fixed risk factors for OSA [6]. There are contradictory views regarding the relationship between alcohol consumption and OSA. 
Some studies [7] suggest that alcohol consumption increases the occurrence of hypoxia and apnea during sleep apnea episodes, but there are also some studies that have not found an increased risk for OSA of alcohol consumption $[8,9]$.

Many people develop OSA after drinking alcohol, particularly before sleep, and there is a high rate of alcohol consumption in China [10], but we still lack studies evaluating the correlation between alcohol consumption and OSA. And there is lack of information about alcohol consumption and OSA in Chinese populations especially; thus, this study will focus on the relationship between alcohol consumption and OSA in patients in China for further discussion.

\section{Materials and methods}

\section{Study population}

This case-control study involved inpatients with suspected OSA admitted from August 2014 to December 2017 to a large Chinese tertiary hospital. The inclusion criteria were (1) inpatients with suspected OSA and (2) inpatients who agreed to have overnight polysomnography (PSG) examination and were able to tolerate it. The exclusion criteria were those (1) could not be reached and (2) did not cooperate with the investigators. The patients were divided into definite
OSA or control groups according to their sleep respiratory monitoring results.

The diagnostic criteria for OSA [11] were as follows: a polysomnographic study with more than 30 episodes of apnea-hypopnea during sleep $7 \mathrm{~h}$ per night or an apnea-hypopnea index (AHI) of $\geq 5$ events/hour.

According to these test results, sleep apnea was either diagnosed or excluded. The patients signed voluntary consent to participate in the sleep respiratory monitoring, which was performed in our hospital respiratory sleep monitoring center from approximately 22:00-22:30 on the first day to the next day at about 5:00 the length of the monitoring time was $7 \mathrm{~h}$. Two doctors who have worked in this field for more than 5 years provided a blinded interpretation of the patients' monitoring results. The other related data were evaluated by a clinical doctor who was unaware of the patients' sleep monitoring results to reduce bias.

All subjects underwent overnight polysomnography (PSG). Detailed records of the sleep respiratory monitoring test results and patient information collected during hospitalization were reliable. The demographic data and accompanying disease information, including age, gender, smoking status, alcohol consumption, height, weight, echocardiographic findings, and other medical conditions, were collected by a trained primary nurse. Age, gender, BMI, coronary heart disease (CHD), and stroke were adjusted
Table 1 Basline characteristics of participants

\begin{tabular}{lllll}
\hline & $N(\%)$ & OSA & & \\
\cline { 4 - 5 } & $n=794$ & No. $(n=108)$ & Yes $(n=688)$ & $P$ \\
\hline Gender & & & & $<0.001$ \\
Male & $614(77.4)$ & $41(38.7)$ & $138(20.1)$ & \\
Female & $179(22.6)$ & $65(61.3)$ & $550(79.9)$ & 0.257 \\
Age(years) & & & & \\
$18-45$ & $124(15.6)$ & $20(18.9)$ & $104(15.1)$ & \\
$46-64$ & $472(59.5)$ & $66(62.3)$ & $407(59.2)$ & 0.214 \\
$\geq 65$ & $197(24.8)$ & $20(18.9)$ & $177(25.7)$ & \\
Coronary heart disease & & & & \\
Yes & $359(45.3)$ & $64(60.4)$ & $371(53.9)$ & 0.244 \\
No & $434(54.7)$ & $42(39.6)$ & $317(46.1)$ & \\
Stroke & & & & \\
Yes & $103(13.0)$ & $96(90.6)$ & $595(86.5)$ & \\
No & $690(87.0)$ & $10(9.4)$ & $93(13.5)$ & \\
BMI & & & & \\
$<18.5$ & $1(0.9)$ & $2(0.3)$ & $0(0.0)$ & \\
$18.5-23.9$ & $16(15.1)$ & $71(10.4)$ & $22(6.0)$ & \\
$24-27.9$ & $46(43.4)$ & $276(40.4)$ & $157(43.1)$ & \\
$\geq 28$ & $43(40.6)$ & $334(48.9)$ & $185(50.8)$ & \\
Drinker & & & & \\
Yes & $365(46.0)$ & $35(33.3)$ & $330(48.0)$ & \\
No & $428(54.0)$ & $70(66.7)$ & $358(52.0)$ & \\
\hline
\end{tabular}


Table 2 Odds ratios (95\% CIs) of OSA for alcohol use in participants

\begin{tabular}{|c|c|c|c|c|c|}
\hline & & $N(\%)$ & $\begin{array}{l}\text { Model A } \\
\text { OR }(95 \% \text { CI })\end{array}$ & $\begin{array}{l}\text { Model B } \\
\text { OR }(95 \% \text { CI })\end{array}$ & $\begin{array}{l}\text { Model C } \\
\text { OR }(95 \% \text { CI })\end{array}$ \\
\hline \multirow[t]{3}{*}{ Alcohol use } & None (reference) & $358(83.6)$ & 1 & 1 & 1 \\
\hline & Yes & $330(90.4)$ & $1.86(1.21-2.87)$ & $2.09(1.34-3.25)$ & $2.03(1.30-3.17)$ \\
\hline & $\mathrm{P}$ & & 0.005 & 0.001 & 0.002 \\
\hline \multirow[t]{4}{*}{ Alcohol exposure group } & None (reference) & $358(83.6)$ & 1 & 1 & 1 \\
\hline & Former & $231(89.9)$ & $1.75(1.08-2.83)$ & $2.01(1.23-3.30)$ & $1.96(1.19-3.22)$ \\
\hline & Current & $99(91.7)$ & $2.18(1.05-4.51)$ & $2.28(1.10-4.73)$ & $2.22(1.06-4.63)$ \\
\hline & P for trend & & 0.005 & 0.001 & 0.002 \\
\hline
\end{tabular}

Model A: crude model, model B: adjusted for age, model C: adjusted for age, BMI, coronary heart disease, and stroke

in multivariate regression analysis. BMI was calculated as weight $(\mathrm{kg}) /$ standing height $(\mathrm{m})^{2}$. CHD and stroke were confirmed by the attending physicians of the Cardiology Department of the large Chinese tertiary hospital according to WHO MONICA diagnostic criteria [12]. As a measure to ensure the accuracy of the information, the patient's answers of the drinking information will be confirmed again by the patient and their relatives.

Alcohol consumption was defined as drinking at least one glass of wine within the past 30 days. According to the World Health Organization (WHO) definition [13], a glass of wine refers to $1 / 2$ a bottle of beer, 2.5 glasses of wines or fruit wine, or 0.8 glasses of wine ( 1 glass is equal to $50 \mathrm{~g}$ ).

\section{Statistical analysis}

IBM SPSS Statistics for Windows, version 24.0, was used for the data analyses. The significance level for all tests was set at a two-tailed $\alpha$ value of 0.05 . The differences in the means and proportions were evaluated using Student's tand chi-square tests, respectively. Logistic regression models were used to identify the association between alcohol consumption on OSA.

\section{Ethical considerations}

This study was conducted in accordance with the Declaration of Helsinki and was approved by the Medical Ethics Committee of the Chinese PLA General Hospital. All participants provided written informed consent before joining the study.

\section{Results}

A total of 793 patients were enrolled in this study, including 688 OSA patients and 106 control subjects. The average age was $57.4 \pm 12.7$ years (range $16-90$ years). The average ages of those who with and without OSA were $57.7 \pm 12.4$ years (range $16-88$ years) and $55.2 \pm 14.1$ years (range 25-90 years), respectively. There were no significant differences in age, coronary heart disease, stroke history, and body mass index between OSA patients and the control group. There were only differences in the sex ratio and alcohol consumption $(P<0.05$, Table 1$)$.

In the logistic regression, we found that, compared with those who did not consume alcohol, those consuming alcohol

Table 3 Odds ratios (95\% CIs) of OSA and hypoxemia for alcohol use in participants

\begin{tabular}{llllll}
\hline & & $\mathrm{N}(\%)$ & $\begin{array}{l}\text { Model A } \\
\text { OR (95\% CI) }\end{array}$ & $\begin{array}{l}\text { Model B } \\
\text { OR (95\% CI) }\end{array}$ & $\begin{array}{l}\text { Model C } \\
\text { OR (95\% CI) }\end{array}$ \\
\hline Alcohol use & & $339(79.2)$ & 1 & 1 & 1 \\
& None (reference) & $321(87.9)$ & $1.93(1.31-2.86)$ & $2.13(1.42-3.18)$ & $2.04(1.36-3.08)$ \\
& Yes & & 0.001 & $<0.001$ & 0.001 \\
Alcohol exposure group & P & $339(79.2)$ & 1 & $2.18(1.38-3.43)$ & $2.09(1.32-3.33)$ \\
& None (reference) & $226(87.9)$ & $1.93(1.24-3.00)$ & $2.02(1.08-3.78)$ & $1.93(1.02-3.64)$ \\
& Former & $95(88.0)$ & $1.94(1.04-3.63)$ & $<.001$ & 0.001 \\
\hline
\end{tabular}

Model A: crude model, model B: adjusted for age, model C: adjusted for age, BMI, coronary heart disease, and stroke 
had a higher risk of OSA (OR 2.03, 95\% CI 1.30-3.17) after adjusting for age, BMI, coronary heart disease, and stroke. Regarding the risk of OSA after adjusting for former drinkers and current ones, the ORs were 1.96 (95\% CI 1.19-3.22, $P<0.05$ ) and 2.22 (95\% CI 1.06-4.63, $P<0.05$ ), respectively. And the $P$ for trend $=0.002$ (Table 2). And we also found that, compared with those who did not consume alcohol, those who did had a higher risk of OSA and hypoxemia (OR 2.04, 95\% CI 1.36-3.08) after adjusting for age, BMI, coronary heart disease, and stroke. Regarding the risk of OSA and hypoxemia after adjusting for former drinkers and current ones, the ORs were similar with ORs for OSA, and the $\mathrm{P}$ for trend $=0.001$ (Table 3).

AHI is the most objective measure of the severity of OSA, so we did the linear regression models to analyze the relationship between alcohol consumption and the AHI in the participants. The result showed that the $\beta$ of former drinkers and the current ones were 3.448 and 4.560 after adjusted for age, BMI, coronary heart disease, and stroke respectively; P for trend was 0.006 (Table 4). And the relationship may have gender difference, alcohol consumption was associated with AHI in female significantly ( $\beta=10.190$ and 15.395 for former and current drinkers respectively in females after adjustment, $\mathrm{P}$ for trend $=0.002$; Table 4).

We also did the logistic regression analysis of Tables 2 and 3 in different gender, and the results were similar (Tables S1 and S2). However, the ORs were not significant in both male and female, and this may due to the reason that few women were drinkers in China for the cultural impact.

\section{Discussion}

In this study, we found that alcohol consumption was an independent risk factor of OSA and OSA with hypoxia, and current drinks had a higher risk of OSA and OSA with hypoxia than former ones. Drinking cessation reduced the increased OSA risk associated with alcohol consumption, but previous alcohol exposure still increases the OSA risk when compared with people who never drink. Alcohol consumption was related to AHI significantly after adjustment, especially in female.

Previous studies showed that the risk factors of OSA are complex; besides obesity, gender, and upper airway anatomical abnormalities, alcohol consumption is an important risk factor [14]. A study of teenagers in Hong Kong showed that drinking not only aggravates sleep difficulties and insomnia, but also makes snoring more pronounced among drinkers [15]. The results of this study suggested that AHI was positively correlated with alcohol consumption, and $\mathrm{LSaO} 2$ was negatively correlated with alcohol consumption, which

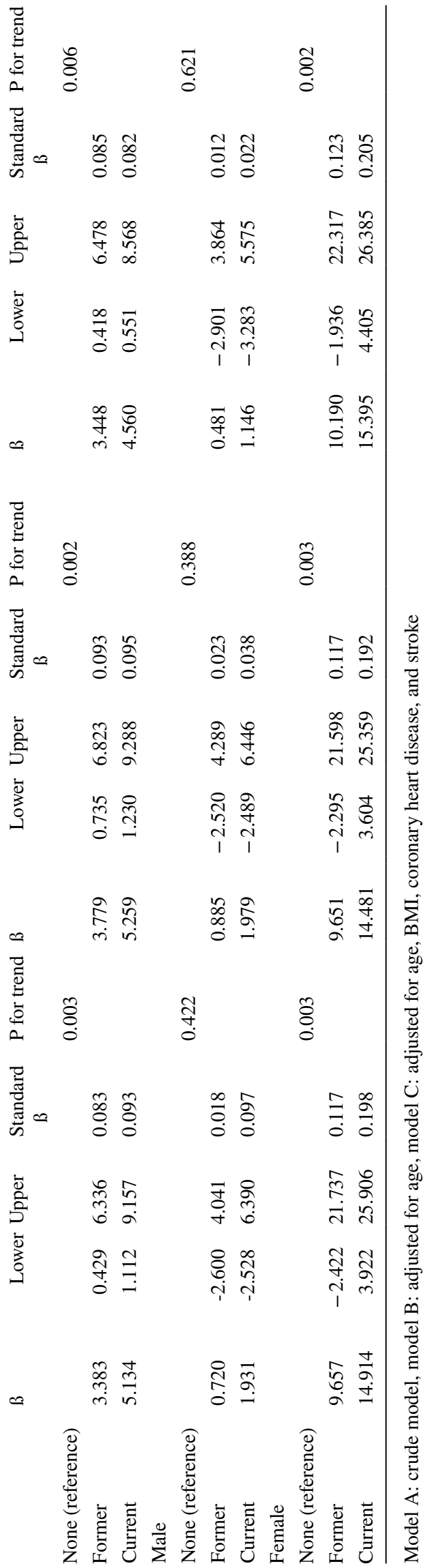

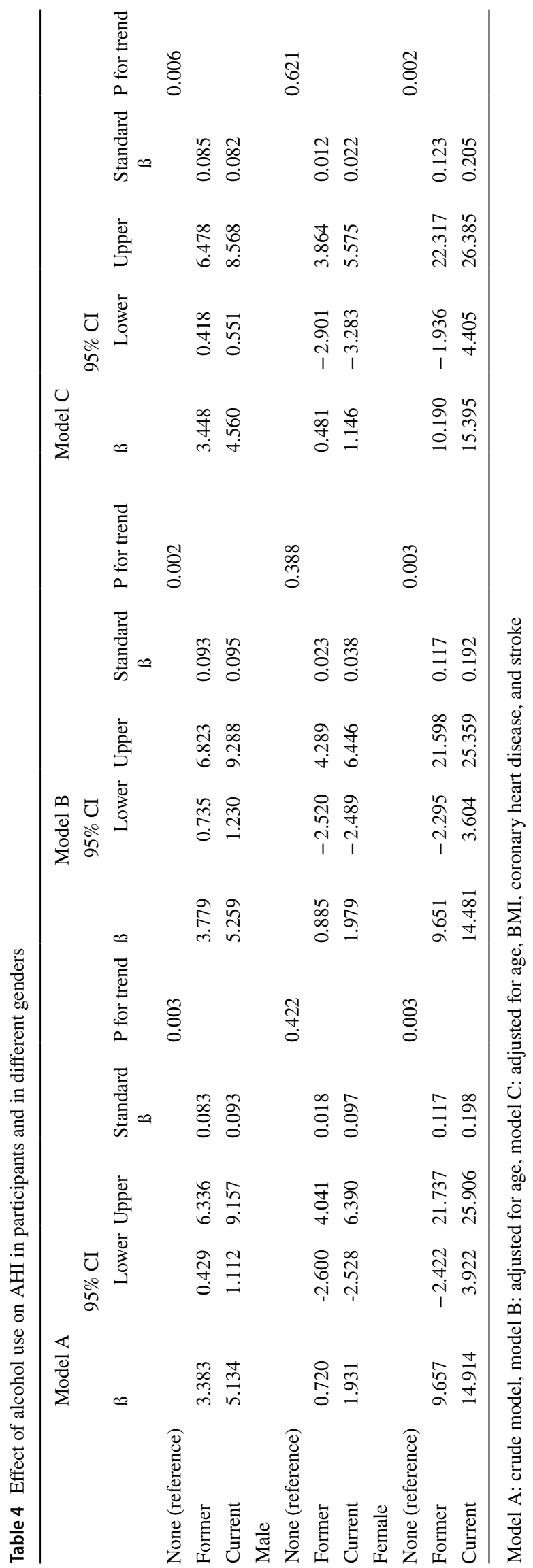


is consistent with the results of Pan Y et al. [16]. And we firstly showed that there was gender difference in the effect of alcohol on OSA and AHI in Chinese population.

The mechanism of OSA induced by alcohol consumption may be related to the collapse of upper airway caused by alcohol consumption [17]. Further, experimental studies have shown that chronic alcohol intake can lead to alveolar type 2 cell damage and alveolar surface glutathione reduction [18]. In ewes exposed to alcohol for a long time, chronic lung injury of fetus, decrease of type 2 cells and SPA on alveolar surface, and decrease of expression of alveolar surface protein $\mathrm{A}$ and $\mathrm{C}$ by one-third [19, 20], these may be the mechanisms of OSA induced by alcohol consumption, but the mechanism of gender differences remains to be further explored.

This study has several limitations. Firstly, this study is a case-control study with inherent memory bias, but we tried to minimize the memory bias by confirming the information of drinking habits to the family members when the progress of the information collection. Secondly, the population in this study is the hospitalized population of a high-level hospital, which is not well represented, and the extrapolation of conclusions needs to be cautious.

\section{Conclusion}

In this study, we found that alcohol consumption was an independent risk factor of OSA and OSA with hypoxia, and alcohol consumption was related with AHI significantly after adjustment, especially in female. In order to reduce the risk and severity of OSA, it is suggested that people should avoid drinking, and drinkers should abstain from drinking.

Supplementary Information The online version contains supplementary material available at https://doi.org/10.1007/s11845-021-02671-7.

Acknowledgements The authors thank Beijing Promise Hui Tong Technology Co. Ltd. for their support in data collection.

Author contribution SSY, YHL, and XHG contributed to data analysis and manuscript writing. SSY, YHL, XHG, and YXLcontributed to study design, data collection, manuscript revision, and approval of the final submission. All authors contributed to the drafting and editing of this manuscript and approved the final version submitted for publication.

Availability of data and materials The datasets used and/or analyzed during the current study available from the corresponding author on reasonable request.

Data availability The datasets used to support this study are not freelyavailable in view of participants' privacy protection.

\section{Declarations}

Ethics approval The study received ethical approval from the Committee for Medical Ethics of the Chinese PLA General Hospital, Beijing, China. All procedures performed in studies involving human participants were also in accordance with the 1964 Helsinki declaration and its later amendments or comparable ethical standards.

Informed consent Informed consent was obtained from all individual participants included in the study.

Conflict of interest The authors declare no competing interests.

Open Access This article is licensed under a Creative Commons Attribution 4.0 International License, which permits use, sharing, adaptation, distribution and reproduction in any medium or format, as long as you give appropriate credit to the original author(s) and the source, provide a link to the Creative Commons licence, and indicate if changes were made. The images or other third party material in this article are included in the article's Creative Commons licence, unless indicated otherwise in a credit line to the material. If material is not included in the article's Creative Commons licence and your intended use is not permitted by statutory regulation or exceeds the permitted use, you will need to obtain permission directly from the copyright holder. To view a copy of this licence, visit http://creativecommons.org/licenses/by/4.0/.

\section{References}

1. Hagen EW, Barnet JH, Hla KM, Palta M, Young T, Peppard PE (2013) Increased prevalence of sleep-disordered breathing in adults. Am J Epidemiol 177:1006-1014

2. Ghazal A, Roghani F, Sadeghi M, Amra B, Kermani-Alghoraishi M (2015) Obstructive sleep apnea, diagnosed by the Berlin questionnaire and association with coronary artery disease severity. ARYA atherosclerosis 11:275-280

3. Morand J, Arnaud C (2018) Chronic intermittent hypoxia promotes myocardial ischemia-related ventricular arrhythmias and sudden cardiac death 8:2997

4. Martins EF, Martinez D, da Silva F, Sezera L, da Rosa de Camargo R, Fiori CZ, et al (2017) Disrupted day-night pattern of cardiovascular death in obstructive sleep apnea. Sleep medicine. 38:144-150

5. Franklin KA, Lindberg E (2015) Obstructive sleep apnea is a common disorder in the population-a review on the epidemiology of sleep apnea. J Thorac Dis 7:1311-1322

6. Deflandre E, Kempeneers D, Degey S, Poirrier R, Legros P, Brichant JF et al (2017) Risk factors for nocturnal hypoxemia in severe obstructive sleep apnea patients. Minerva Anestesiol 83:449-456

7. Baik I, Seo HS, Yoon D, Kim SH, Shin C (2015) Associations of sleep apnea, NRG1 polymorphisms, alcohol consumption, and cerebral white matter hyperintensities: analysis with genome-wide association data. Sleep 38:1137-1143

8. Coughlin SR, Mawdsley L, Mugarza JA, Calverley PM, Wilding JP (2004) Obstructive sleep apnoea is independently associated with an increased prevalence of metabolic syndrome. Eur Heart $\mathbf{J}$ 25:735-741

9. Sharafkhaneh A, Giray N, Richardson P, Young T, Hirshkowitz M (2005) Association of psychiatric disorders and sleep apnea in a large cohort. Sleep 28:1405-1411

10. Feng Y, Newman IM. (2016) Estimate of adolescent alcohol use in China: a meta-analysis. Archives of public health $=$ Archives belges de sante publique. $74-45$ 
11. Capener DC, Brock MS, Hansen SL, Matsangas P, Mysliwiec V. (2018) An initial report of sleep disorders in women in the U.S. Military. Military Medicine

12. Truelsen T, Mähönen M, Tolonen H, Asplund K, Vanuzzo D (2003) Trends in stroke and coronary heart disease in the WHO MONICA Project. Stroke 34:1346-1352

13. Lurie A (2011) Obstructive sleep apnea in adults: epidemiology, clinical presentation, and treatment options. Adv Cardiol 46:1-42

14. Damián M, Fernando SG, José Antonio R, María GG, Pedro C, Cristina N, et al (2005) Factors with influence on sleep apnea/ hypopnea syndrome. Med Clin. 125: 681-684

15. Huang R, Ho D, Lo W, Lai H, Lam T (2013) Alcohol consumption and sleep problems in Hong Kong adolescents. Sleep Med 14:877-882

16. Pan Y, Wang W, Wang KS. (2014) Associations of alcohol consumption and chronic diseases with sleep apnea among US adults. Int J High Risk Behav Addict. 3: e19088
17. Bikov A, Hull JH, Kunos L (2016) Exhaled breath analysis, a simple tool to study the pathophysiology of obstructive sleep apnoea. Sleep Med Rev 27:1-8

18. Freddy R, Dilip S, Michelle D, William S, Hoek JB, Kallen CB et al (2014) Chronic alcohol ingestion in rats alters lung metabolism, promotes lipid accumulation, and impairs alveolar macrophage functions. Am J Respir Cell Mol Biol 51:840-849

19. Lazic T, Wyatt TA, Matic M, Meyerholz DK, Grubor B, Gallup JM et al (2007) Maternal alcohol ingestion reduces surfactant protein A expression by preterm fetal lung epithelia. Alcohol 41:347-355

20. Foula S, Luke OD, Gert M, Kelly K, Victoria S, Nadine B et al (2009) Repeated ethanol exposure during late gestation alters the maturation and innate immune status of the ovine fetal lung. Ajp Lung Cellular \& Molecular Physiology 296:510-518

Publisher's Note Springer Nature remains neutral with regard to jurisdictional claims in published maps and institutional affiliations. 